\title{
Investigating the feasibility of MePlusMe, an online intervention to support mental health, wellbeing, and study skills in higher education students
}

\author{
Rhianna Goozee ${ }^{1}$, Alexia Barrable ${ }^{2}$, Jelena Lubenko ${ }^{3}$, Marietta Papadatou-Pastou ${ }^{4}$, \\ Mark Haddad $^{5}$, Eamonn McKeown ${ }^{5}$, Shashivadan P Hirani ${ }^{5}$, Maryanne Martin ${ }^{6}$, and \\ Patapia Tzotzoli ${ }^{7}$
}

${ }^{1}$ Independent scholar, UK

${ }^{2}$ University of Dundee, School of Education and Social Work, UK

${ }^{3}$ Department of Health Psychology and Paedagogy, Rīga Stradiṇš University, Latvia

${ }^{4}$ School of Education, National and Kapodistrian University of Athens, Greece

${ }^{5}$ School of Health Sciences, City, University of London, UK

${ }^{6}$ Department of Experimental Psychology, University of Oxford, UK

${ }^{7}$ My Psychology Clinic \& iConcipio Ltd, UK

Corresponding author:

Ass. Prof. Marietta Papadatou-Pastou

National and Kapodistrian University of Athens, Greece

Tel: +30 6932398693;

Email: marietta.papadatou-pastou@seh.oxon.org

Draft version 1.0, 2.4.2021. This paper has not been peer reviewed. Please do not copy or cite without author's permission. 
ONLINE INTERVENTION FEASIBILITY STUDY 


\begin{abstract}
Introduction: While there are several web-based interventions to support mental health, few target students in higher education (HE). Importantly, more research is needed to establish their effectiveness. Here, we provide a pragmatic evaluation of an online intervention (MePlusMe) specifically designed to improve the mental health, wellbeing, and study skills of HE students.

Methods: In accordance with the published protocol for a feasibility study, we obtained a convenience sample of $137 \mathrm{HE}$ students, with students recruited to participate in an 8-week intervention. To evaluate the feasibility and assess the effects of this online intervention, validated measures of mood (depression and anxiety), wellbeing and self-efficacy were collected at baseline, 2, 4 and 8 weeks, alongside two feedback forms assessing design and functionality (baseline), and engagement (week 4 and 8).
\end{abstract}

Results: We observed statistically significant reductions in levels of anxiety and depression, and increases in wellbeing over the 8-week intervention period. However, there were no significant changes in self-efficacy. Participants rated the system design and functionality positively, and qualitative findings indicated high levels of satisfaction with MePlusMe. Some areas for improvement were also identified.

Discussion: Rates of initial consent, subsequent engagement with the programme, and measure completion, together with qualitative feedback, support the acceptability of the intervention. Significant benefits were observed for the main intended outcomes, with the overall results providing qualified support for the effectiveness of MePlusMe. The significant positive changes associated with use of this system are encouraging. Nonetheless, modest retention rates limit the precision and generalisability of these findings. Further investigation should ascertain optimal duration of engagement, most acceptable means of outcome assessment, and further detail about obstacles to utilisation. 
Keywords

Students, digital intervention, higher education institutions, mental health, COVID-19 


\section{Introduction}

Around three quarters of individuals with a lifetime mental health disorder experience their first episode by 25 years old. ${ }^{1}$ Moreover, becoming a student at a higher education institution (HEI) constitutes a major transition and entails several challenges that can further exacerbate or even trigger the onset of mental health issues..$^{2-4}$ It is therefore unsurprising that many higher education (HE) students worldwide experience mental health difficulties, including depression, anxiety, and other mental health and substance abuse disorders. ${ }^{4-8}$ Anxiety and depression are common among HE students in the US, ${ }^{2} \mathrm{UK}^{7}$ and further afield. ${ }^{5} \mathrm{~A}$ recent large-scale international survey of 14,000 students conducted by the World Health Organisation (WHO) reported 12-month prevalence rates of 17\% (generalised anxiety disorder) and 19\% (major depressive episode). ${ }^{9}$

Mental health difficulties can have a negative effect on several aspects of university and personal life, including reduced engagement with campus life, impaired academic performance, and lower graduation rates. ${ }^{8,10,11}$ Importantly, students may experience reduced quality of life. ${ }^{12}$ Elevated prevalence of suicide ideation in HE students is also evident, ${ }^{10,13}$ and could lead to higher levels of suicide attempts and death by suicide. ${ }^{14}$

Despite reported levels of both clinical and subclinical psychological disorders, few students receive treatment. According to a US study, only $15 \%$ of students experiencing moderate or severe depression received treatment, and less than $16 \%$ of those with suicide ideation were receiving help. ${ }^{15}$ There may be many reasons students do not seek help, with US students reporting that they view stress as a normal part of studying in HE, they wish to deal with stress alone, and they do not have time for treatment. ${ }^{16}$

The stigma of mental illness also plays a role. Students may be subject to personal or public stigma, with certain characteristics, such as race or religiosity, determining the level of stigma experienced. ${ }^{16}$ Higher personal stigma has been significantly associated with lower 
levels of help-seeking in a sample of over 5,500 college students. ${ }^{16}$ Moreover, male and minority students may be among those least likely to seek help for mental health problems due to stigma. ${ }^{16,17}$

Student support services (SSS) exist onsite to address and manage students' mental health difficulties. There is increasing demand for such services, which SSS may struggle to meet due to lack of funding. Moreover, several further barriers that prevent students accessing SSS have been identified, including stigma (as discussed above), lack of time, and concerns about confidentiality. ${ }^{18}$ Finally, the recent COVID-19 pandemic has highlighted the need for undisrupted support in unprecedented times. Internet-based support systems could provide a solution to many of these difficulties by offering cost-effective support that can be accessed 24/7. ${ }^{19}$ Moreover, such interventions may be particularly well-suited to HE students who are unlikely to seek help elsewhere. ${ }^{20}$ Internet-based systems can also provide support for mild to moderate difficulties allowing SSS to focus their limited resources on severe cases.

There has been a recent proliferation of online support systems, with increasing evidence supporting their use for mental health difficulties in various populations. ${ }^{21-25}$ According to a recent review, among the 16 online support systems available to students, six were specifically designed for HE students, of which just four have been subjected to empirical research to determine their effectiveness. ${ }^{26}$ Therefore, further research is necessary to establish the efficacy and effectiveness of online support systems in student populations.

Here, we report the results of a feasibility study of a proposed online support system, called MePlusMe, which is being developed specifically for HE students. In this study, we tested a simulation of the system to assess effects of its use and inform the next stage of its development. 


\section{Methods}

\section{Study design and procedure}

The current study was based on methods outlined in a previously published protocol, with some necessary deviations due to sample size. ${ }^{27} \mathrm{~A}$ within-subjects repeated measures study was conducted between October 2018 and February 2019, with students at Rīga Stradiņ̌s University, Latvia. The study received ethical approval from the research ethics committee of Rīga Stradiņš University (Nr.6-3/1).

Participants were recruited via an advertisement sent out through the university's formal information channels (email, posters, and the official social media account). Interested students completed initial self-report questions to identify severe difficulties and risk factors such as self-harm, substance abuse, and unusual sensory experiences or beliefs. Any students reporting these indicators were excluded from the study and immediately provided with clear details of HEI services and other sources of support (see protocol for full risk screening detail $\left.{ }^{27}\right)$. Students reporting no difficulties, or mild to moderate psychological and/or academic-related difficulties were eligible for inclusion.

Included participants completed demographic and baseline measures online before attending a group study day at which the MePlusMe system and one example video technique were presented, followed by an offline simulation of the system. Students completed the initial Questionnaire or Library form (see MePlusMe below) to inform the selection of appropriate video techniques, which were then provided digitally for further practice at home in the subsequent weeks.

Following the initial presentation, students provided feedback on the design and functionality of the system, and video format of the techniques. Outcome measures were subsequently obtained at weeks 2,4 , and 8 . At weeks 4 and 8 , students also provided further feedback regarding their engagement with the video techniques and potential effects of use. 
MePlusMe

MePlusMe is a web-based support system designed to support HE students experiencing mild to moderate psychological difficulties or academic difficulties, and students without difficulties wanting to learn how to take care of their mental health and improve their study skills. It can be used alone or to complement traditional support services (for example, while waiting to receive support or following intervention). The final system will assess the risk of severe mental health difficulties and where relevant, signpost students to more appropriate sources of support.

Following risk assessment, eligible students will access videos with psycho-education and techniques via two routes, a symptoms-route ('Questionnaire'; in which identified symptoms are linked to recommended techniques) or a techniques-route ('Library'; where users choose which techniques they wish to learn). As they practise techniques over time, users can monitor their progress with responses to a single item global function question 'How are you getting on now in your daily life?'.

Motivational reminders and facilitated social networking will also be built into the system to encourage engagement and mutual support ('MyPlace'). Moreover, embedded filters identify any users who develop more significant difficulties and refer them to seek further support from SSS or primary care. MePlusMe has been rigorously developed, founded on current best practice and evidence-based techniques to ensure the system is fit for purpose.$^{28}$ Further details regarding the system and its development have been published elsewhere. ${ }^{26-30}$

\section{Clinical measures}


At baseline and at weeks 2, 4 and 8, students completed the Patient Health Questionnaire (PHQ-9), ${ }^{31}$ Generalized Anxiety Disorder Scale (GAD-7), ${ }^{32}$ Warwick-Edinburgh Mental Wellbeing scale (WEMWB), ${ }^{33}$ and Academic Self-Efficacy Scale (SEF). ${ }^{34}$

The PHQ-9 is a validated measure of depressive symptoms, widely used in primary care and general populations including HE students. It has nine items scored on four-point Likert scales ranging from 'not at all' (scoring "0”) to 'nearly every day' (scoring “3”). Total scores range from 0 to 27 , with higher scores indicating greater severity of depressive symptoms.

The GAD-7 is a well-validated measure of generalized anxiety. It has seven items scored on four-point Likert scales ranging from 'not at all' (scoring "0") to 'nearly every day' (scoring “" 3 ”). Total scores range from 0 to 21 , with higher scores indicating greater severity of anxiety symptoms.

The WEMWB was developed to measure mental wellbeing among the general population with an initial validation sample comprising university students together with general survey participants. It has 14 items scored on five-point Likert scales ranging from 'none of the time' (scoring "1") to 'all of the time' (scoring "5"). Total scores range from 14 to 70 , with higher scores indicating better mental wellbeing.

The original SEF developed with US students comprises 27 statements describing positive study-related behaviours scored on a 10-point scale ('not at all confident' scoring “0” to 'extremely confident' scoring "10"). This scale was adapted (with permission) to: (i) adapt wording to the European setting (e.g., "term papers" replaced by “coursework") and (ii) remove items with limited relevance ("Having more tests in the same week" and "Getting along with family members"). Total self-efficacy scores ranged from 0 to 250 , with higher scores indicating greater self-efficacy. 


\section{Open-ended responses and satisfaction ratings}

In addition to clinical measures, two feedback forms were administered, with open-ended questions, scaled satisfaction items ('liked' ... 'disliked'; 'poor' ... 'very good'), and a question about how participants are getting on in their daily life (everyday functionality), rated from 1 ('extremely well') to 7 ('not well at all'). At the initial assessment, questions were asked on system design, general functionality, and video format. Further questions were administered at weeks 4 and 8 on system use, perceived benefits and user satisfaction.

\section{Data analysis}

Descriptive statistics (means and standard deviations or standard errors) are reported for sample characteristics and outcomes. Linear mixed model (LMM) analyses were conducted with SPSS v25 to examine differences in scores between time points for each outcome. LMM analyses were subsequently conducted to explore the main effects of time and demographic/academic factors (e.g., gender, qualification level etc.), and their potential interactions (significance set at $p=0.05$ ). Where interaction effects were significant, post hoc tests were conducted within time points and within each level of a factor to decompose effects (with Sidak's correction for multiple comparisons ${ }^{35}$ ). Satisfaction ratings were examined with descriptive analyses. Coding of the open-ended text responses was carried out independently by two members of the research team, and the key themes to be reported were then discussed and agreed. ${ }^{36}$ 


\section{Results}

\section{Sample characteristics}

Following recruitment, 137 students were enrolled in the study (mean age: $22.6( \pm 3.38)$ years; male: $n=39$; female: $n=98)$. The majority of participants were EU students $(n=76$, $55.5 \%)$, while $32.1 \%(n=44)$ were home students, and $12.4 \%(n=17)$ were international students. Most participants $(60 \%)$ were in their first $(n=33)$ or second $(n=36)$ year of study. Following initial recruitment, 68 participants were retained at week 2, 45 at week 4, and 26 at week 8 . Given this high dropout rate, LMM analyses were conducted to retain as much data as possible (Table 1).

\section{[Insert Table 1]}

\section{Clinical measures}

There was a significant reduction in anxiety across the eight weeks $\left(F_{(3,53.26)}=9.57, p<.001\right)$, with baseline anxiety levels significantly higher than at all follow-up time points. There was also a significant reduction in depression across the intervention period $(F(3,55.82)=4.94$, $p=.004$ ), with analysis at individual timepoints showing the greatest reduction by week 2 , with non-significant changes in later weeks. Wellbeing scores steadily increased across time $\left(F_{(3,46.99)}=3.04, p=.038\right)$, with significantly greater scores at week 8 than at baseline. The only measure not showing significant change was academic self-efficacy.

\section{[Insert Figure 1]}

$L M M$ analyses were also conducted to determine the effect on the results of gender, qualification level, status/ethnicity, study program, accommodation, and whether participants 
opted for reminders. There were no significant interactions for most variables, indicating they did not influence changes in outcomes over time.

Only the provision of reminders had a significant interaction effect with time on mental wellbeing and self-efficacy scores (see Table 1). While there were no significant differences between mental wellbeing scores at each time point for those who received reminders and those who did not, there were significantly greater scores at week 8 (mean $=$ $54.95, S E=3.86)$ than at week $4($ mean $=44.45, S E=3.15)$ in the no-reminder group. Despite a significant interaction effect of the provision of reminders over time on selfefficacy scores, no significant differences were indicated by post hoc tests (either within time-point, or within-groups).

[Insert Figure 2]

[Insert Figure 3]

\section{Use and impressions of the system}

Reasons for participation. Participants gave various reasons for taking part in the study, with $39 \%(n=54)$ reporting study difficulties and 33\% $(n=45)$ reporting emotional or psychological difficulties. However, the most common reason for participating in the study simply wanting to find out more about MePlusMe $(61 \%, n=83)$.

System design. Participants rated various design features of the 'Questionnaire', 'Library', 'MyPlace,' and video techniques on a five-point scale from 'very poor' to 'very good'. The 'Questionnaire', 'Library', and 'MyPlace' were all found to be easy to use and useful, with clear wording (rated by over $83 \%$ of participants as 'good' or 'very good'). Responses to 
open-ended questions supported this, however areas identified for improvement included a lack of relevance to their personal situation and limited or over-simplified content.

Both the illustrations and narrator's voice in the videos were considered 'good' or 'very good' by the majority of participants ( $84 \%$ and $89 \%$, respectively). The storyboard and ability to maintain interest were also positively rated ('good' or 'very good' by $95 \%$ and $85.5 \%$ of participants, respectively). Open-ended questions suggested that participants thought the videos and animations were high quality and the voices were soothing but negative aspects included that videos were not high definition, and the introduction cannot be skipped.

Table 2 provides an overview of some of the strengths and areas for potential improvement in terms of system design reported by participants. As a whole, the system was reported to be clear, with an attractive colour scheme. Positive aspects of functionality included the ability to personalise content. The archiving and reminders features were seen as useful and several participants commented that being an online system enhanced the user experience. A few areas for further development and improvement were identified. Some participants commented that the colour scheme was unengaging and the interface was plain, while some illustrations were poorly produced. In terms of functionality, negative comments related to an overly complicated design and low resolution of videos.

The written and video content was found by participants to be positive, relevant, and motivational. They found that content helped them deal with their emotions and that goals set were realistic. The main concern regarding content was that it was too generic, with the questionnaire being singled out as not identifying certain feelings and having somewhat irrelevant questions, as well as being too long-winded.

[Insert Table 2] 
Use of the system. At week 4, the largest proportion of students reported having only watched the videos once. However, the frequency increased by week 8 , with $69 \%$ watching them 1-3 times each month. Frequency of practising techniques reduced over time, with most participants reporting a frequency of 1-3 times a week at week 4 and 1-3 times a month at week 8. Various reasons were reported for low frequency of use, including lack of time or not finding the videos or techniques personally relevant or useful. However, some participants found they could use the techniques as needed and did not need to revisit the videos as they had already internalised the content.

Most participants reported they did not access other types of support (for either feelings or study skills support) during the study, with reasons including being too busy or fear of being judged by others. However, at week 8 , all those who did not seek help reported this was because it was not needed. Most participants reported that if they needed support in the future they would seek help from university resources, as well as MePlusMe.

Perceived benefits. In addition to clinical measures, participants were asked if they had experienced any change in their feelings or study skills across the study. The majority reported a perceived change in how they felt at both week 4 and 8 , with improvements such as feeling more confident, optimistic, relaxed and rational, as well as having fewer breakdowns. However, for some there was an increased pressure to study and they felt more stressed. At week 4, only half of the participants reported a change in study skills, but by week 8 the majority reported a change. Improvements included increased concentration and motivation, as well as better time management.

Over the duration of the study, there were improvements in the participant's reports of their overall function, as identified by responses to the question about how they were getting 
on with their daily life. At baseline, a minority of participants (14\%) reported function at the highest levels ('extremely well' or 'very well'), rising to a third by week 4, and half of the participants at week 8 .

Satisfaction. The majority of participants were 'satisfied' or 'very satisfied' with the system at all timepoints. Reasons for satisfaction included the system being straightforward to use, having learned some good techniques via the videos, and improved time management. Dissatisfied participants reported that the system was too simplistic for complex problems or not relevant to their current situation. At 4 and 8 weeks, the majority of participants also said they were either 'likely' or 'very likely' to recommend the system to a friend and were planning to continue using it. Reasons included the benefits they had experienced and that it was likely to help others. For those who were not sure whether they would continue use, lack of time, forgetfulness and already knowing the techniques were cited as reasons.

Suggested improvements. Finally, participants were asked to suggest any possible improvements for the overall system. Overarching improvements included more specific information on study techniques and managing stress/mood, as well as targeting students early in their university career and including more techniques. In terms of functionality, improvements included better design, better animations, more interactivity, and increased personalisation. Further difficulties that participants suggested the system could address included homesickness, social skills, and dealing with family expectations. 


\section{Discussion}

The present study investigated an online mental health and study skills intervention, MePlusMe, for students attending HEI. MePlusMe is a web-based support system designed to support HE students experiencing mild to moderate psychological difficulties, as well as academic difficulties. Anxiety and depression are common in HE students worldwide, $2,5,7,9$ and negatively affect university and personal life in several ways. ${ }^{8,10,11}$ However, helpseeking appears to be low among students, with the majority not receiving treatment. ${ }^{15,16}$ Internet-based support systems such as MePlusMe may overcome some of the barriers to help-seeking faced by students, by offering a cost-effective source of confidential support that can be accessed 24/7. Such systems also provide students with continuing access to remote support, which may be useful during times in which face-to-face support may be less available, as has been experienced during the recent COVID-19 pandemic.

While several online systems are available to support mental health and wellbeing, there remains a need for empirically supported interventions designed specifically for HE students. ${ }^{26}$ MePlusMe is being designed with this in mind and the current feasibility study forms part of a body of research ${ }^{27-30}$ being gathered to support the effectiveness and acceptability of the system among its intended audience. The 8-week intervention was wellreceived, with results indicating a significant positive effect of its continued use. Across the intervention period, there were significant improvements in validated measures of anxiety, depression, and mental wellbeing. Changes in these factors were not influenced by participant characteristics such as gender, qualification level, ethnicity etc. In addition, participants reported a range of positive outcomes in response to open-ended questions, with initial views of the intervention being generally positive.

At baseline, mean scores for anxiety and depression were within the 'mild' range (5 mild; 10 - moderate; 15 - severe). There was a small but significant decrease in depression 
scores over time, the mean anxiety score at final follow up was below the 'mild' cut off (GAD-7 score at week 8, 4.80 \pm 0.71 ). As well as improvement in anxiety and depression symptoms, we identified positive changes in participant's wellbeing. MePlusMe targets common psychological and emotional problems at lower levels of severity, such as mild to moderate depression and mixed anxiety and depressive symptoms. In contrast, other systems designed for HE students have been designed for more severe cases, which makes it difficult to compare results directly. Nonetheless, our finding that the intervention reduced anxiety and depression is similar to studies of these systems. ${ }^{37,38}$

Despite improvements in mood and wellbeing, we did not find any statistically significant improvements in self-efficacy associated with system use. It may be that there are further unmeasured confounding or moderating variables (such as academic performance and feedback) that are relevant here. The extent of changes that we identified indicate that our study was under-powered to identify change in this variable.

This study not only addressed potential effectiveness of the MePlusMe simulation in improving symptoms of psychological problems and their impact, but also investigated the acceptability of MePlusMe in terms of layout, functionality and content through open-ended questions. Overall, responses were positive for all these areas, while students also indicated some areas for development. Students' answers indicated that the system was easy to use, well laid out, and relevant. These responses were further supported by ratings of the layout, content, and usefulness of system features, content including the library of techniques and the video techniques. High ratings (of 'good' or 'very good') were given by the majority of users for the layout and ease of navigation of the 'Library', the video techniques, and the clarity of phrasing. Moreover, $87 \%$ rated the overall layout and ease of navigation of the system as 'very good' or 'good'. These ratings suggest that students were highly satisfied with the system in terms of functionality, content, and layout. 
Engagement and adherence among users is a particular area of concern if web-based interventions are to be useful. While users completing the current study indicated high satisfaction with MePlusMe, there was, unfortunately, a high attrition rate. This reflects a recognised difficulty in engaging university students in mental health interventions. ${ }^{39}$ There are several possible explanations for dropout rates in the current study. Possibly, the length or format of the intervention did not appeal to all students. In addition, all participants had access to the video techniques regardless of their continued participation in the study. Therefore, it cannot be known whether or not participants who failed to complete follow up measures were still using the videos but were not motivated to provide follow up feedback.

\section{Future directions}

Given the increasing burden of psychological difficulties among HE students, there is a need to develop solutions that can overcome some of the barriers to access for those seeking support. Online interventions are one such solution and our data supports the potential for MePlusMe, an online intervention designed specifically for HE students, to lead to positive improvements in outcomes among a diverse HE student population. An improved study design, with a control group and initial randomisation would yield more robust evidence for efficacy.

\section{Conclusion}

In this study, we observed significant positive changes in clinical measures associated with use of a simulation of MePlusMe, an online intervention specifically designed for students. Moreover, qualitative data suggest that initial views of the intervention were generally positive. Despite the study's limitations, these results provide qualified but positive support 
for the feasibility and effectiveness of MePlusMe, and can be used to further inform its future development. 


\section{Acknowledgements}

This article received writing support from Dr Rhianna Goozee, a freelance writer and editor. All authors have contributed to the writing of the paper and authorize the submission of the final manuscript.

\section{Declaration of conflicting interests}

PT is the CEO \& Founder of iConcipio Ltd. No other authors have any conflicting interests. 


\section{References}

1. Kessler RC, Amminger GP, Aguilar-Gaxiola S, et al. Age of onset of mental disorders: a review of recent literature. Curr Opin Psychiatry 2007; 20(4): 359-364. Available from: https://doi.org/10.1097/YCO.0b013e32816ebc8c.

2. Pedrelli $P$, Nyer $M$, Yeung $A$, et al. College students: mental health problems and treatment considerations. Acad Psychiatry 2015; 39(5): 503-511. Available from: http://dx.doi.org/10.1007/s40596-014-0205-9.

3. Ibrahim AK, Kelly SJ, Adams C, et al. A systematic review of studies of depression prevalence in university students. J Psychiatr Res 2013; 47(3): 391-400. Available from: https://doi.org/10.1016/j.jpsychires.2012.11.015.

4. Blanco $C$, Okuda $M$, Wright $C$, et al. Mental health of college students and their noncollege-attending peers: results from the national epidemiologic study on alcohol and related conditions. Arch Gen Psychiatry 2008; 65(12): 1429-1437. Available from: https://doi.org/10.1001/archpsyc.65.12.1429.

5. Yi S, Peltzer K, Pengpid S, et al. Prevalence and associated factors of illicit drug use among university students in the association of southeast Asian nations (ASEAN). Subst Abuse Treat Prev Policy 2017; 12: 9. Available from: https://doi.org/10.1186/s13011017-0096-3.

6. Bennett TH, Holloway KR. Drug use among college and university students: findings from a national survey. J Subst Use 2015; 20: 50-55. Available from: https://doi.org/10.3109/14659891.2013.878762.

7. Andrews $B$, Wilding JM. The relation of depression and anxiety to life-stress and achievement in students. Br J Psychol 2004; 95: 509-521. Available from: https://doi.org/10.1348/0007126042369802.

8. Auerbach RP, Mortier P, Bruffaerts R, et al. Mental disorder comorbidity and suicidal thoughts and behaviors in the World Health Organization World Mental Health Surveys International College Student initiative. Int J Methods Psych Res 2019; 28(2): e1752. Available from: https://dx.doi.org/10.1002\%2Fmpr.1752.

9. Auerbach RP, Mortier P, Bruffaerts R, et al. The WHO World Mental Health Surveys International College Student Project: Prevalence and Distribution of Mental Disorders. J Abnorm Psychol 2018; 127(7): 623-638. Available from: https://doi.org/10.1037/abn0000362.

10. Garlow SJ, Rosenberg J, Moore JD, et al. Depression, desperation, and suicidal ideation in college students: results from the American Foundation for Suicide Prevention College Screening Project at Emory University. Depress Anxiety 2008; 25(6): 482488. Available from: https://doi.org/10.1002/da.20321.

11. Downs MF, Eisenberg D. Help seeking and treatment use among suicidal college students. J Am Coll Health 2012; 60(2): 104-114. Available from: https://doi.org/10.1080/07448481.2011.619611.

12. Pillay N, Ramlall S, Burns JK. Spirituality, depression and quality of life in medical students in KwaZulu-Natal. South Afr J Psych 2016; 22(1): 731. Available from: https://dx.doi.org/10.4102\%2Fsajpsychiatry.v22i1.731.

13. Cukrowicz KC, Schlegel EF, Smith PN, et al. Suicide Ideation Among College Students Evidencing Subclinical Depression. J Am Coll Health 2011; 59: 575-581. Available from: https://doi.org/10.1080/07448481.2010.483710.

14. Garlow SJ, Rosenberg J, Moore JD, et al. Depression, desperation, and suicidal ideation in college students: results from the American Foundation for Suicide Prevention 
College Screening Project at Emory University. Depress Anxiety 2008; 25: 482-488. Available from: https://doi.org/10.1002/da.20321.

15. Eisenberg D, Hunt J, Speer $\mathrm{N}$ et al. Mental health service utilization among college students in the United States. J Nerv Ment Dis 2011; 199: 301-308. Available from: https://doi.org/10.1097/nmd.0b013e3182175123.

16. Eisenberg D, Downs MF, Golberstein E, et al. Stigma and help seeking for mental health among college students. Med Care Res Rev 2009; 66: 522-541. Available from: https://doi.org/10.1177/1077558709335173.

17. Loya F, Reddy R, Hinshaw SP. Mental illness stigma as a mediator of differences in Caucasian and South Asian college students' attitudes toward psychological counseling. J Couns Psychol 2010; 57: 484-490. Available from: https://psycnet.apa.org/doi/10.1037/a0021113.

18. Givens JL, Tjia J. Depressed medical students' use of mental health services and barriers to use. Acad Med 2002; 77: 918-921. Available from: https://doi.org/10.1097/00001888-200209000-00024.

19. Andersson G, Titov N. Advantages and limitations of Internet-based interventions for common mental disorders. World Psychiatry 2014; 13: 4-11. Available from: https://doi.org/10.1002/wps.20083.

20. Ryan ML, Shochet IM, Stallman HM. Universal online interventions might engage psychologically distressed university students who are unlikely to seek formal help. Adv Ment Health 2010; 9: 73-83. Available from: https://doi.org/10.5172/jamh.9.1.73

21. Stratton E, Lampit A, Choi I, et al. Effectiveness of eHealth interventions for reducing mental health conditions in employees: a systematic review and meta-analysis. PLoS ONE 2017; 12: e0189904. Available from: https://doi.org/10.1371/journal.pone.0189904.

22. Schaub MP, Blankers $M$, Lehr $D$, et al. Efficacy of an internet-based self-help intervention to reduce co-occurring alcohol misuse and depression symptoms in adults: study protocol of a three-arm randomised controlled trial. BMJ Open 2016; 6: e011457. Available from: https://doi.org/10.1136/bmjopen-2016-011457.

23. Ebert DD, Cuijpers $P$, Muñoz RF, et al. Prevention of mental health disorders using internet- and mobile-based interventions: a narrative review and recommendations for future research. Front Psychiatry 2017; 8: 116. Available from: https://doi.org/10.3389/fpsyt.2017.00116.

24. Andersson G, Carlbring P, Ljótsson B, et al. Guided internet-based CBT for common mental disorders. J Contemp Psychother 2013; 43: 223-233. Available from: https://doi.org/10.1007/s10879-013-9237-9.

25. Christensen H, Batterham $P$, Mackinnon A, et al. Prevention of generalized anxiety disorder using a web intervention, iChill: randomized controlled trial. J Med Internet Res 2014; 16: e199. Available from: https://doi.org/10.2196/jmir.3507.

26. Papadatou-Pastou M, Goozee R, Payne E, et al. A review of web-based support systems for students in higher education. Int J Ment Health Syst 2017; 11: 59. Available from: https://doi.org/10.1186/s13033-017-0165-z.

27. Papadatou-Pastou M, Goozee R, Barley EA, et al. Online intervention, 'MePlusMe', supporting mood, wellbeing, study skills, and everyday functioning in students in higher education: a protocol for a feasibility study. Pilot Feasibility Stud 2015; 1: 34. Available from: http://dx.doi.org/10.1186/s40814-015-0029-8. 
28. Barrable A, Papadatou-Pastou M, Tzotzoli P. Supporting mental health, wellbeing and study skills in Higher Education: an online intervention system. Int J Ment Health Syst 2018; 12: 54. Available from: https://doi.org/10.1186/s13033-018-0233-z.

29. Papadatou-Pastou M, Campbell-Thomson L, Barley E, et al. Exploring the feasibility and acceptability of the contents, design, and functionalities of an online intervention promoting mental health, wellbeing, and study skills in Higher Education students. Int J Ment Health Syst 2019; 13: 51. Available from: https://doi.org/10.1186/s13033019-0308-5.

30. Touloumakos AK, Goozee RM, Papadatou-Pastou M, et al. Online support system for students in higher education: proof of concept study. Digit Health 2016; 2: 2055207616655012. Available from: https://dx.doi.org/10.1177\%2F2055207616655012.

31. Spitzer RL, Williams JBW, Kroenke K, et al. Validity and utility of the PRIME-MD Patient Health Questionnaire in assessment of 3000 obstetric-gynecologic patients: the PRIME-MD Patient Health Questionnaire Obstetrics-Gynecology Study. Am J Obstet Gynecol 2000; 183: 759-769. Available from: https://doi.org/10.1067/mob.2000.106580.

32. Spitzer RL, Kroenke K, Williams JBW, et al. A brief measure for assessing Generalized Anxiety Disorder. The GAD-7. Arch Intern Med 2006; 166(10): 1092-1097. Available from: https://doi.org/10.1001/archinte.166.10.1092.

33. Tennant R, Hiller L, Fishwick R, et al. The Warwick-Edinburgh Mental Well-Being Scale (WEMWBS): development and UK validations. Health Qual Life Outcomes 2007; 5: 63. Available from: https://dx.doi.org/10.1186\%2F1477-7525-5-63.

34. Zajacova A, Lynch SM, Espenshade TJ. Self-efficacy, stress, and academic success in college. Res High Educ 2005;46(6):677-706. Available from: https://doi.org/10.1007/s1116 2-004-4139-z.

35. Šidák ZK. Rectangular confidence regions for the means of multivariate normal distributions. J Am Stat Assoc 1967; 62(318): 626-633. Available from: https://doi.org/10.1080/01621459.1967.10482935.

36. Smith CP. Content analysis and narrative analysis. In: Reis HT, Judd CM, editors. Handbook of research methods in social and personality psychology. Cambridge: Cambridge University Press; 2000. p. 313-335.

37. Lynch S, Gander ML, Kohls N, et al. Mindfulness-based coping with university life: a nonrandomized wait-list-controlled pilot evaluation. Stress Heal 2011; 27: 365-375. Available from: https://doi.org/10.1177\%2F2158244018758379.

38. Musiat $P$, Conrod $P$, Treasure J, et al. Targeted prevention of common mental health disorders in university students: randomised controlled trial of a transdiagnostic trait-focused web-based intervention. PLoS ONE 2014; 9(4): 1-10. Available from: https://doi.org/10.1371/journal.pone.0093621.

39. Melville KM, Casey LM, Kavanagh DJ. Dropout from Internet-based treatment for psychological disorders. Br J Clin Psychol. 2010; 49(Pt 4): 455-471. Available from: https://doi.org/10.1348/014466509x472138. 


\section{Tables and Figures}

Table 1. Linear mixed models analyses showing changes in each outcome over time

\begin{tabular}{lllllllll}
\hline & \multicolumn{2}{l}{ Time (weeks) } & \multicolumn{4}{c}{ Effect of time } \\
\cline { 2 - 8 } Outcome (mean \pm SE) & 0 & 2 & 4 & 8 & $\mathrm{DF}_{1}$ & $\mathrm{DF}_{2}$ & $\mathrm{~F}$ & $P$ \\
\hline Anxiety & $8.23^{\mathrm{a}} \pm 0.37$ & $6.28^{\mathrm{b}} \pm 0.44$ & $6.29^{\mathrm{b}} \pm 0.52$ & $4.80^{\mathrm{b}} \pm 0.71$ & 3 & 53.26 & 9.57 & $<0.001$ \\
\hline Depression & $8.49^{\mathrm{a}} \pm 0.42$ & $6.92^{\mathrm{b}} \pm 0.46$ & $7.45^{\mathrm{a}, \mathrm{b}} \pm 0.73$ & $6.55^{\mathrm{a}, \mathrm{b}} \pm 0.82$ & 3 & 55.82 & 4.94 & 0.004 \\
\hline Wellbeing & $45.13^{\mathrm{a}} \pm 0.73$ & $\begin{array}{l}46.49^{\mathrm{a}, \mathrm{b}} \pm \\
0.98\end{array}$ & $\begin{array}{l}47.24^{\mathrm{a}, \mathrm{b}} \pm \\
1.29\end{array}$ & $49.97^{\mathrm{b}} \pm 1.62$ & 3 & 46.99 & 3.04 & 0.038 \\
\hline Academic self-efficacy & $178.54 \pm$ & $\begin{array}{l}182.38 \pm \\
4.44\end{array}$ & $\begin{array}{l}185.43 \pm \\
6.05\end{array}$ & $\begin{array}{l}196.57 \pm \\
7.43\end{array}$ & 3 & 45.96 & 2.11 & 0.112 \\
\hline
\end{tabular}

For each analysis, superscript letters indicate scores at each time point that are significantly different. 
Table 2. Perceived strengths and areas of improvement identified by participants

\section{Strengths}

Layout

I thought it was designed in a clear way to find out exactly what my main problems are and how to help me with them (Participant 29)

Well thought out concept, which includes every aspect of a student's life (Participant 119)

Simple layout, ability to tailor needs (Participant 15)

It is made so that it feel as if you are in your personal space, your little room (Participant 35)

\section{Functionality}

It is quite easy to navigate through the portal. I like that it is kept simple without much distraction (Participant 41)

This is a very good way to personalize help for students and their needs for a support (Participant 72)

I like how you can personalize it according to your liking (Participant 59)

It seems very straightforward (Participant 44)

\section{Content}

Just pure and easily understandable information that actually matches perfectly what I was looking for to cope with stress (Participant 1)

Clarity of the information make[s] it useful for anyone (Participant 94)

Specific features have names that makes it easy to understand what it is about so the clarity of the phrasing is understandable (Participant 34)

I like that they have videos with solutions to almost anything that one might experience during university (Participant 81)

\section{Areas for improvement}

The website is a little old fashioned in its design and could benefit from being perhaps more modern, particularly as your target group is students (Participant 105)

The layout seemed a bit stiff and kind of too much emphasized studying (Participant 4)

A more modern, clearer and minimalistic website design (Participant 68) It is fair and one would find it useful [...], but user experience really matters, so the design and layout shows room for improvement. More user friendly, more images instead of words as well. People rather have expressions than words (Participant 83)

Make it more interactive. If you're giving students study techniques, follow through and have them do things on the site to keep them engaged (Participant 43)

Terminology is kept rather general, which makes it quite hard to specify what is actually meant. More selection possibilities should be possible (Participant 57)

Had a good selection but I feel like it could have more alternatives (Participant 63)

Options can be elaborated a bit more, more relaxation techniques can be added, more user-initiated program structure that pushes the participant to record and build progress, and feel like he/she is improving using the system (Participant 19) 
Making it more interesting - making real situations, not animations. Looks sometimes too childish (Participant 54) 


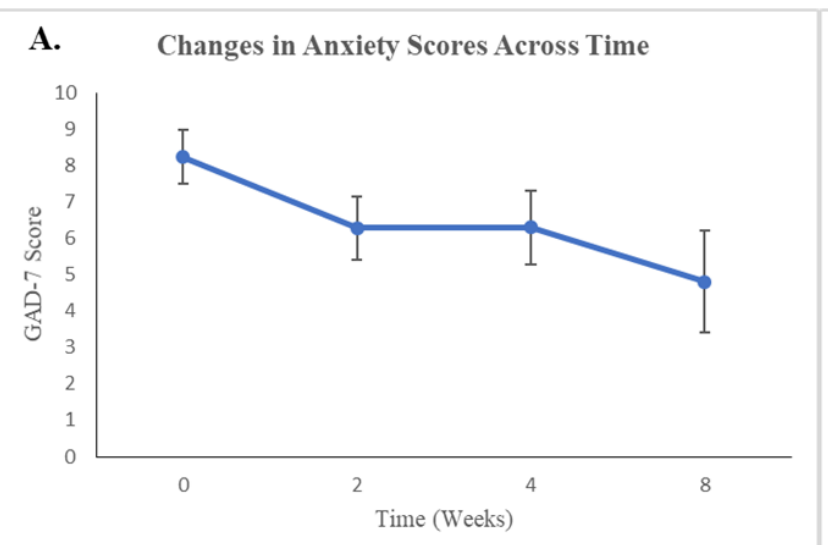

C. Changes in Mental Wellbeing Across Time

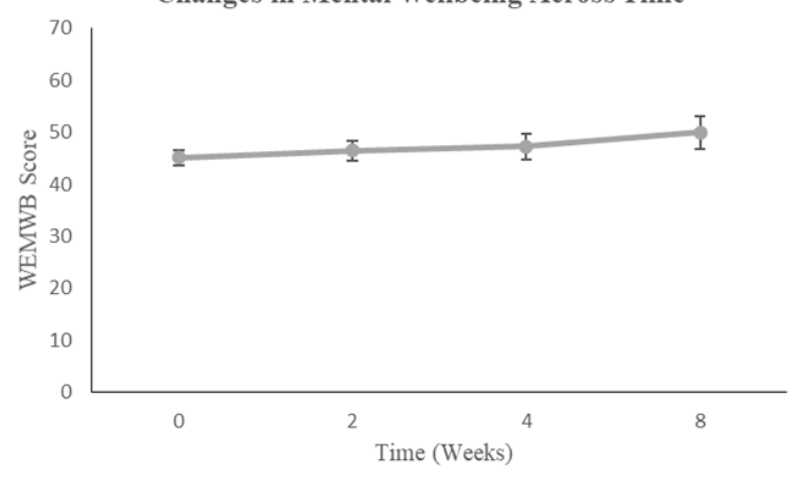

B. Changes in Depression Scores Across Time

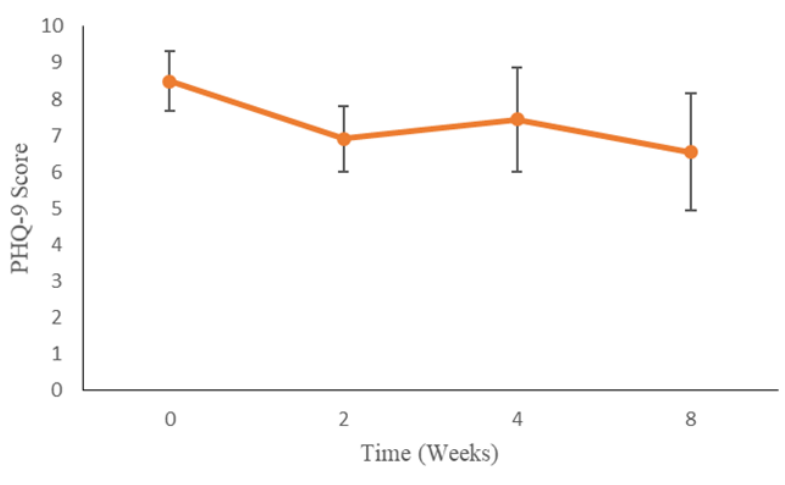

D. Changes in Self Efficacy Scores Across Time

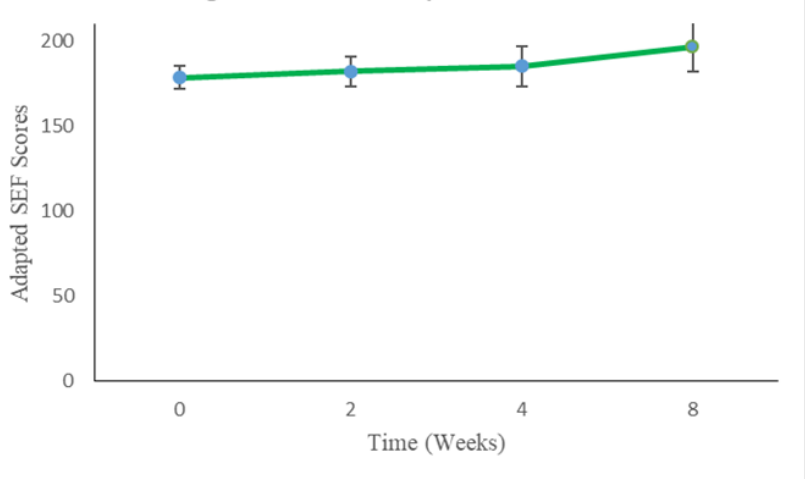

Figure 1. Changes in anxiety (A), depression (B), mental wellbeing (C) and self-efficacy (D) over 8 weeks of treatment.

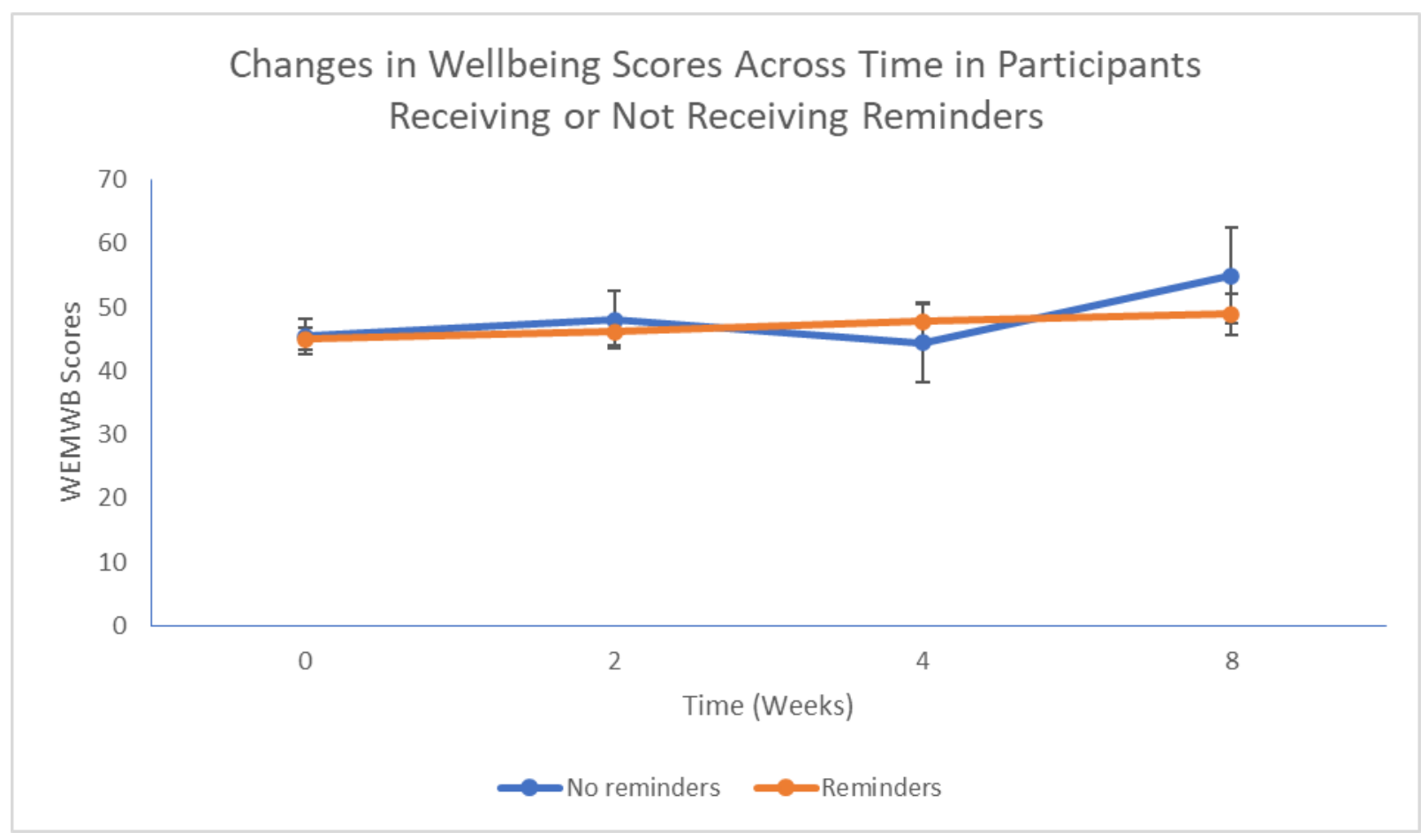


Figure 2. Changes in mental wellbeing scores over time for participants receiving or not receiving reminders.

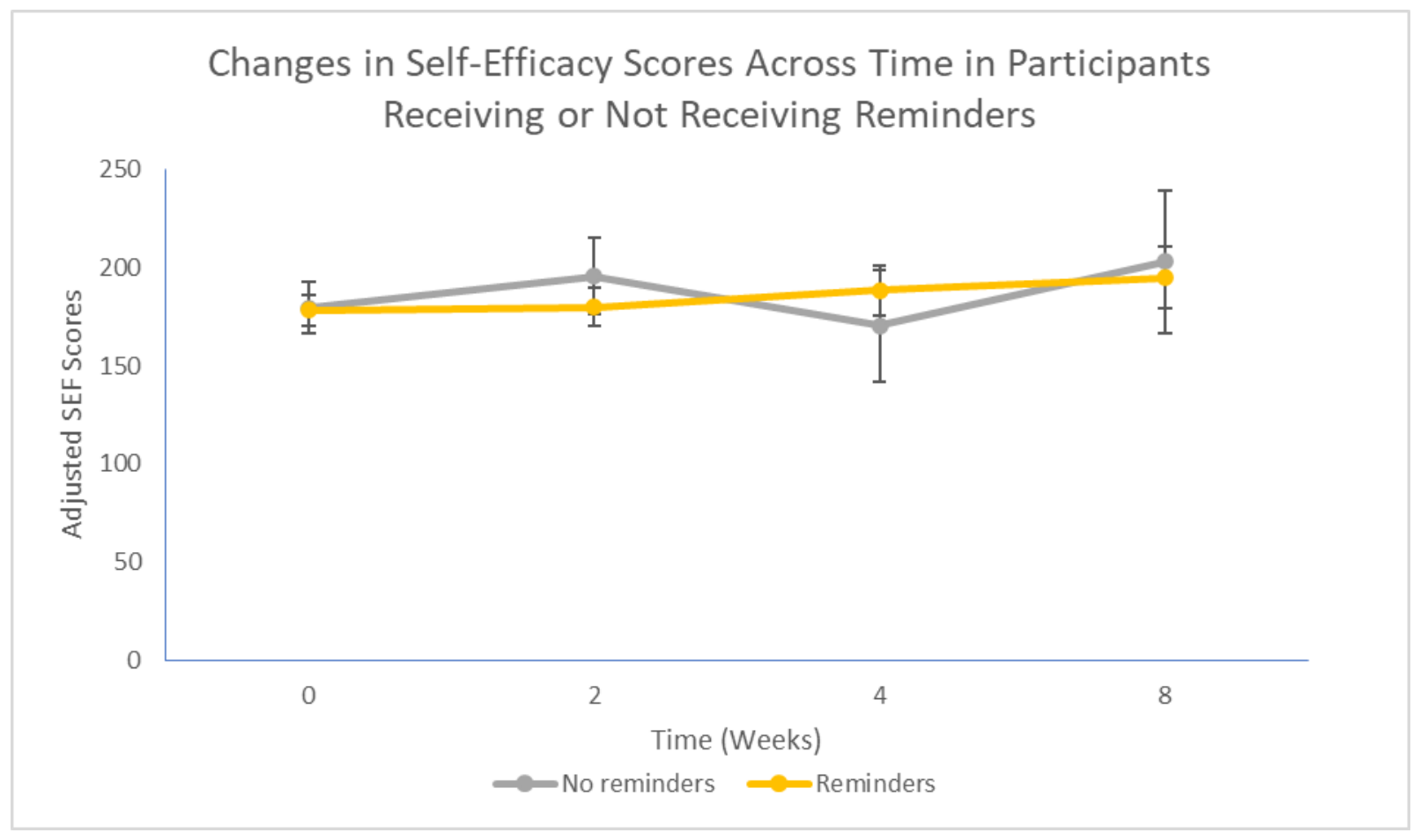

Figure 3. Changes in self-efficacy scores over time for participants receiving or not receiving reminders. 